\title{
Emergence and Consequences of Lateral Sample Heterogeneity in Glow Discharge Spectrometry
}

Andrew P. Storey ${ }^{1}$, Steven J. Ray ${ }^{1,2}$, Volker Hoffmann ${ }^{3}$, Maxim Voronov ${ }^{3}$, Carsten Engelhard ${ }^{1,4}$, Wolfgang Buscher ${ }^{5}$, Gary M. Hieftje ${ }^{1 *}$

1 Indiana University, Department of Chemistry, 800 E. Kirkwood Ave., Bloomington, IN 47405, USA

2 Department of Chemistry, University at Buffalo, The State University of New York, Buffalo, NY 14260

3 IFW Dresden e.V., Leibniz Institute for Solid State and Materials Research Dresden, POB 270116, 01171 Dresden, Germany

4 University of Siegen, Department of Chemistry and Biology, Adolf-ReichweinStr. 2, D-57076 Siegen, Germany

5 University of Münster, Department of Inorganic and Analytical Chemistry, Corrensstr. 30, D-48149 Münster, Germany

*Corresponding author: hieftje@indiana.edu

\section{Research Paper for Submission to Spectrochimica Acta, Part B}

\section{Keywords:}

Glow discharge

Atomic emission spectrometry

Depth profiling

Sample morphology 


\section{Abstract}

Conventional glow discharge emission or mass spectrometry requires the assumption that the surface of the sample is homogeneous. However, recent developments in glow discharge imaging appear to offer an opportunity to obtain three-dimensional concentration maps, in which this assumption is no longer necessary. Here, experiments, models, and a summary of earlier work are combined to examine the sputtering behavior of elemental and morphological heterogeneities in a sample. The theoretical model reveals gaps in current knowledge of glow discharge sputtering of heterogeneous samples, particularly indicating that heterogeneity in the sample leads to roughened crater bottoms and how additional morphology can evolve. Additionally, a three-dimensional profiling microscope is used to characterize the effects of surface inclusions on the sputtering process in a DC glow discharge in a reduced-pressure argon environment. Findings have important implications for bulk analysis, depthprofiling, and elemental surface mapping with glow discharge spectrometry.

\section{Introduction}

Quantitative depth profiling with glow-discharge (GD) sources is used with both optical spectroscopy and mass spectrometry for the elemental characterization of thin films, ${ }^{1}$ semiconductors, ${ }^{2}$ and several other classes of materials. $^{3}$ In depth-profiling applications, it is necessary to determine the sputtering rate of the sample to determine the depth corresponding to each subsequent spectrum. ${ }^{4,5}$ In turn, the rate of sputtering is largely derived from 
Equation 1, which was described by Boumans in the early 1970's, during the early days of glow-discharge spectrometry. ${ }^{4,5}$

$$
Q=C_{Q} \cdot \dot{1}_{g} \cdot\left(V_{g}-V_{0}\right) \quad \text { (Equation 1) }
$$

In this equation, $Q$ is the sputtering rate and $V_{g}$ and $i_{g}$ are the discharge potential and current, respectively. The other two variables $-C_{Q}$ and $V_{0}-$ are dependent on the material of the cathode and the discharge support gas. The threshold voltage, $V_{0}$, is a minimal potential required to sustain the discharge and induce sputtering: ${ }^{6}$

$$
V_{0}=\frac{\left(m_{i}+m_{t}\right)^{2}}{4 m_{i} m_{t}} U_{0} \quad \text { (Equation 2) }
$$

Variables $m_{i}$ and $m_{t}$ are the masses of incident and target particles and $U_{0}$ is the heat of sublimation for each material. The material-dependent sputtering constant, $C_{Q}$ (with units of $\mu \mathrm{g} \cdot \mathrm{W}^{-1} \cdot \mathrm{s}^{-1}$ ), is empirically observed to be related to the atomic weight and the heat of sublimation of the sample also, but this term is not entirely predictable, especially for multi-element samples.

Preferential sputtering (more rapid removal of some elements than others in the same sample) within macroscopically homogeneous samples has been described previously. ${ }^{7,8}$ The principles behind these earlier studies have enabled quantitative mass spectral and emission measurements of bulk materials that have components with different sputtering rates. When GD spectrometry is used for bulk analysis, a pre-burn period is employed, which establishes an equilibrium of surface concentrations before the analytical measurement is begun. ${ }^{8}$

Flat-bottomed craters are generally presumed to indicate even sputtering across the surface of a sample. ${ }^{9,10}$ However, finer observation commonly reveals 
a roughened surface on the crater bottom that can arise from microscopic heterogeneity within a sample. Disparity in the sputtered depth across the surface of a sample is a greater problem when macroscopic inclusions are present, which results in variation in the depths and sputtering rates between entire regions of a sample. Glow discharge depth-profiling methods typically employ algorithms that use the composition of the sample to estimate the sputtering rate. ${ }^{11-13}$ A necessary assumption of these techniques is that the elements observed in the spectra are homogeneously distributed about the lateral dimensions of the sampled area. ${ }^{14}$ However, this assumption is not always valid; some materials have laterally heterogeneous structure ${ }^{15}$ and others have unexpected inclusions ${ }^{16}$ that can alter the characteristics of the material.

Several researchers have described problems that arise from sample heterogeneity in glow discharge spectrometry. In the early 1970s, Dogan, Massman, and Laqua ${ }^{7}$ observed the difference in the height of two materials in the same sample that sputtered at different rates. They expanded this study with electron micrographs that revealed topographic structures in the crater bottom and described how variations in sample composition ultimately resulted in changes in the I-V characteristics of the source and in the associated sputtering behavior. Their work addressed both microscopic heterogeneity in alloyed materials and how these differences were likely to translate to macroscopic variations. $^{7}$ Later, Weiss ${ }^{8}$ described the sputtering-based enrichment of carbon on the surface of graphitic steel and explained some of the associated quantitative calibration techniques. 
Over the past decade, several optical techniques have been developed to view spatially resolved emission across the surface of glow discharge samples. ${ }^{17-}$ 20 These techniques utilize pulsed glow discharges in 0.1 to $4.0 \mathrm{kPa}$ of argon to temporally and spatially isolate emitting species near where they were sputtered from the surface. Higher pressures shrink the mean free path, which limits the lateral diffusion of atoms in the time between their removal from a sample surface and emission they yield in the negative glow region of the discharge. ${ }^{18}$ These techniques generally provide lateral spatial resolution on the order of hundreds of micrometers, making them appropriate for the characterization of macroscopic variation across the surface. Ideally, spatially resolved measurements would be coupled with depth-profiling techniques to produce a three-dimensional map of a sample. However, this goal can be achieved only with a full understanding of how lateral variation affects the sputtering process.

Other surface-analysis techniques (e.g. Auger electron spectroscopy, ${ }^{21} \mathrm{x}$ ray photoelectron spectroscopy, ${ }^{22}$ and secondary ion mass spectrometry ${ }^{23}$ ) offer three-dimensional spatial resolution, but generally do so by sputtering and sampling small parts of the surface sequentially. ${ }^{21-23}$ Due to this point-by-point approach, high-resolution images can require many hours to raster across a sample. Glow discharge spectrometry offers the advantage over these other surface-analysis techniques of simultaneously sputtering away an entire surface layer of the sample, enabling a much faster analysis. ${ }^{24}$ However, simultaneous sputtering creates a complication for heterogeneous samples, especially when quantification of both concentration and depth are desired. 
Several aspects of depth-profile GD analysis can limit precision and accuracy. Instrumental conditions can play a role if redeposition occurs, ${ }^{25}$ electric fields are uneven, ${ }^{26}$ or conditions in the discharge change ${ }^{26}$. Interfacial effects can also be important if the transition between layers is large enough. ${ }^{27}$ In such situations, errors from earlier layers are propagated through the sampling process such that each subsequent interface appears broadened. ${ }^{28}$ The paucity of high-quality layered standards is also limiting in many cases.

Of course, depth quantification is further complicated by the need to convert emission signal to concentration ( $y$-axis of a depth profile) and time to depth (x-axis). This two-part quantitation problem has been described in detail elsewhere. ${ }^{29}$ Additionally, variation at a sample interface can be dealt with mathematically to deconvolve unevenness that can evolve at such interfaces. ${ }^{30}$ However, all these methods currently require the assumption that the layers are generally homogeneous.

The present study focuses on challenges for GD depth profiling that arise because of the expectation of laterally homogeneous samples and how observations of intentionally heterogeneous samples provide insight into how heterogeneity in GD samples is manifested in an analytical signal. These studies include an examination of how theory that assumes lateral homogeneity can be affected by sample inclusions. Experimental results indicate also the manner in which heterogeneities and surface morphology affect signals from both the minority inclusion and majority constituent of the sample. 


\section{Materials and Methods}

2.1 Simulation program. The simulation program was written in LabVIEW ${ }^{\mathrm{TM}} 14$ (National Instruments, Austin, TX). The operational principles behind the simulation's operation can be found in Figure 1; the program itself is available as electronic supplementary information.

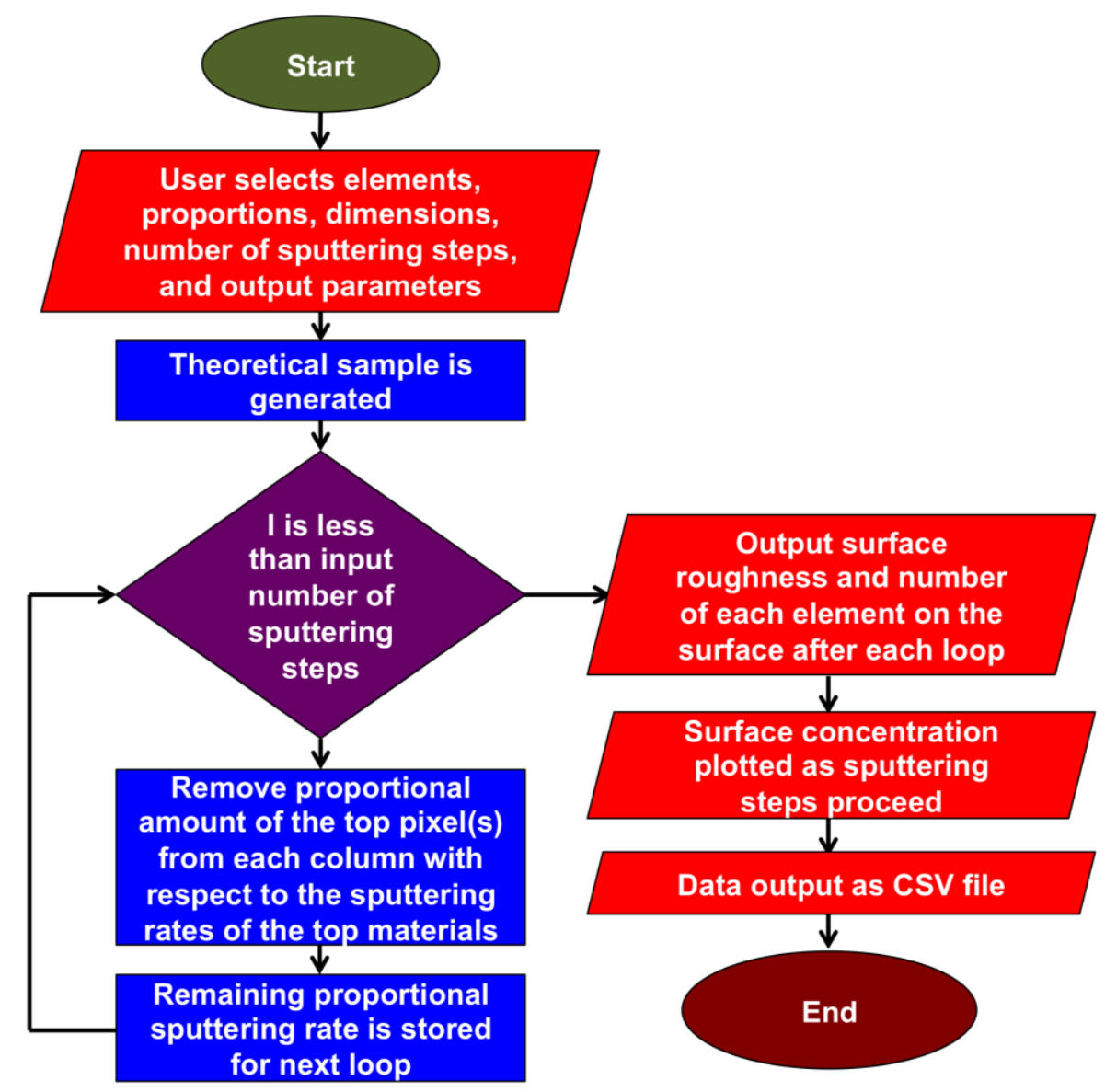

Figure 1: Flow chart of essential operations of the sputtering-simulation program. Operational program can be found in electronic supplemental material.

2.2 Sample preparation. Simulated metal inclusions were inserted into samples by drilling small holes of the same diameter as a piece of the selected metal wire. Care was taken to ensure that the holes extended only part way through the 
sample. A small segment of wire was then pressed into each hole after which the sample was ground flat. All samples were ground and polished with $4 / 0$ grit polishing paper before analysis. Copper ( $1 \mathrm{~mm}$, bare copper wire, $\mathrm{p} / \mathrm{n} 155452 \mathrm{~A}$, Fisher Brand), aluminum (1 mm, 99.999\% Al, Alfa Aesar, p/n MFCD00134029), and silver wire (2 mm, 99.9\% Ag, Acros Organics, $\mathrm{p} / \mathrm{n}$ 317720010) were all inserted as inclusions in brass. Microscopic examination of these inclusions found no observable gaps between the materials before they were sputtered with the glow discharge. A scratched brass sample was created by dragging a stainless steel screw across the brass surface after grinding and polishing.

2.3 Profiling microscopy. An optical profiling microscope (Zeta 20, Zeta Instruments, San Jose, CA) was used to obtain three-dimensional true-color images of samples before and after sputtering. With the 50x objective used for these measurements, the optical resolution was $0.42 \mu \mathrm{m}$ with an achievable vertical resolution of $100 \mathrm{~nm}$. For the measurements here, 300 vertical steps were used over the range of the measurement (typically on the order of 30 to 45 $\mu \mathrm{m})$.

2.4 Glow discharge operating parameters. The glow-discharge cell used for these studies analyzed a 9-mm diameter area of each sample. A customfabricated cell was used that has been described previously. ${ }^{31}$ A DC power supply (Fluke Programmable High Voltage Power Supply, model 4150A, Everett, WA, USA) was used for these experiments, with current limited to $20 \mathrm{~mA}$ at a pressure of $0.13 \mathrm{kPa}$ of argon. For the measurement of a silver pin sputtered at the edge of a sample, sputtering was allowed to progress for 4 hours . Later experiments with silver, copper, and aluminum inclusions in the bottom of the 
crater and the scratched surface were sputtered for 2 hours under the same conditions, except with a Spellman DC power supply (p/n X2957, Hauppauge, NY, USA).

2.5 Time-dependence measurement. A sample was sputtered in 30-min intervals over a total of 3 hours to measure the change in depth during the sputtering process. The sample was replaced after each measurement with the Zeta-20 microscope. The Fluke power supply was used for these measurements.

\section{Theory}

\subsection{Program to Model GD Sputtering of Heterogeneous Materials}

A program to model sputtering of heterogeneous samples was written that enables preferential erosion of different materials to be visually represented. Figure 1 shows a flow chart of how this program functions. The program generates a sample with a selected set of characteristics, including randomly dispersed pure elements and their respective percentages in the sample. A number of sputtering steps (I in Figure 1) is then generated to remove material based on the relative sputtering rate of each constituent. This process can be repeated to collect statistics on the behavior of different samples of the same bulk composition but varied initial atomic location. A simple version of this program can be found in the electronic supplementary information.

An example of how this model performs can be seen in Figures $2 \mathrm{~A}$ and $2 \mathrm{~B}$ in which a sample comprised of carbon and iron atoms leads to a great deal of surface roughness during the sputtering process. This program assumes perpendicular ion attack and removal of atoms from a simple, computer-gridded 
sample. This behavior is true to the ideal creation of flat-bottomed craters from laterally homogeneous samples, which are required for accurate depth profiling in conventional glow-discharge spectrometry ${ }^{10}$. Nevertheless, simulated sputtering results in a notably uneven sputtered surface, as shown in Figure 2B.

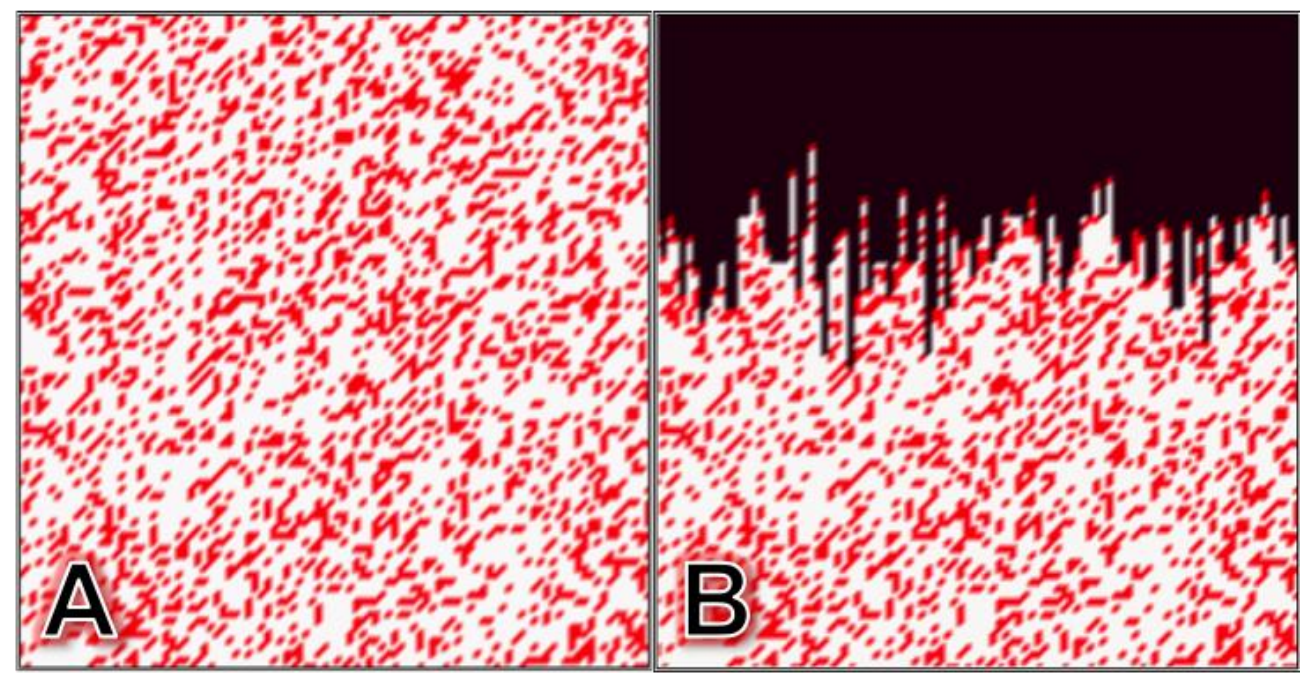

Figure 2: Side (depth) view of computer model for newly generated sample (A) and its surface after sputtering (B) for a hypothetical specimen that is $75 \%$ iron (white pixels) and 25\% carbon (red pixels). Black region in B represents the portion that has already been sputtered away. Depletion of iron on the surface results in a much lower average surface concentration than in the bulk material.

If this material-dependent variation were the extent of the complication, the resulting roughness would be predictable from physical properties of the sample and could be used to approximate the contribution of heterogeneity to signal variations. However, the sputtering process is known to be more complicated, ${ }^{32}$ which highlights the limitations of such a simple model and 
conventional assumptions of glow discharge spectrometry. The non-idealities are discussed in more detail later.

The selection of iron and carbon in Figure 2 is representative of graphitized cast iron, a material that is expected to exhibit microscopic heterogeneity and mimics the samples described by Weiss. ${ }^{8}$ Iron (white pixels in Figure 2) is depleted and carbon (red pixels) is enriched on the surface almost immediately due to the lower sputtering rate of carbon. For a sample in which $\mathrm{Fe}$ and $\mathrm{C}$ are randomly distributed, this enrichment would ideally occur during the "pre-burn" period that is common for glow discharge bulk analysis. After the "preburn", the higher overall concentration of accessible carbon at the surface would largely compensate for its lower sputtering rate. For bulk analysis, these variations will all eventually equilibrate to yield a representative measurement. However, if a concentration gradient were present for graphite in iron, changes as sputtering progresses could be missed. Of course, in many samples, other complications can arise; Weiss ${ }^{8}$ noted in his work with graphitized cast iron that grinding such samples can pull out flakes of graphite, which could artificially enrich initial iron concentration on the surface.

In the present model, dimensions are not specified, but it is useful to consider how a range of dimensions, from pixels representing individual atoms to visible macroscopic inclusions, might produce dissimilar effects. Our discussion begins with microscopic inclusions, which are considered in earlier studies ${ }^{7,8}$ of heterogeneity and are most relevant to conventional GD analyses. In the case where pixels of the program represent very tiny areas (e.g. single atoms), an 
argon ion would need to penetrate perpendicularly to reach the bottom of a deep crevice without being pulled toward one of the very near and oppositely charged walls that surround its path. Similarly, for an ejected atom to make it out of the bottom of such a narrow well, it would need to traverse upward the same confined path in order to be excited in the negative glow. This behavior is extremely unlikely, but the effects of such a process, in which an impinging ion would be directed toward that lower depth, would include sputtering the side wall that had developed in the sample, not just the top. Thus, at small dimensions, elevated regions are expected to experience greater sputtering rates than low areas that are nearby. This situation pertains to the graphitized carbon example of Figure 2.

Additionally, atoms ejected from a sample surface are not necessarily those that are directly impacted by the incident species, and the arrangement of the impacted surface could change as the result of redeposition ${ }^{33}$ and surface mixing ${ }^{28}$. When an incident ion or neutral atom strikes the surface, it sets off a collisional cascade, resulting in ejection of a nearby atom when the energy from the impact event (or series of events) is greater than the atom binding energy. This behavior will still result in the more easily sputtered atoms leaving the sample surface first; however, extreme differences in surface depth likely will not propagate the cascade through the sample efficiently. Ziegler has described the effects of microscopic sample texture on attenuating or stopping this cascade. ${ }^{34}$

Although earlier work focused largely on the effect of heterogeneities on bulk analysis, preferential sputtering perhaps has an even greater impact when 
depth profiling is employed. Surface enrichment and depletion of particular elements can occur in each layer of a depth-profile measurement; for thin layers, there will not be sufficient time for equilibration to occur. In a multi-layered sample, this lack of equilibration apparent in our model and previous studies ${ }^{8}$ would propagate into successive layers. Further, constituents of a given layer would not always have the same time-dependent signal behavior. More rapidly sputtering elements would likely produce an initial signal peak followed by a decline to a stable level. In contrast, slower sputtering elements should yield a shallower signal slope overall but level out at a time similar to that of the fastersputtering element.

When preferential sputtering occurs in the top portion of a sample, it often affects behavior at the interface between underlying layers. In such situations, the signal from a faster-sputtering element present in an underlying layer could rise slowly because its atoms are covered by a slower-sputtering one. Depending upon the nature of the underlying layer, this action might extend the overlap between layers in a depth profile. These features appear to be present in many glow discharge depth profiles, including recently published quantitative depth-profiling measurements. ${ }^{35-37}$

In simulations of more sizable heterogeneities, single pixels would represent micron dimensions or greater. This type of situation is largely unstudied; such macroscopic heterogeneity would violate the assumptions necessary for depth profiling. In this scenario, an argon ion (or a fast neutral) might be capable of reaching the bottom of a deep well that had previously been 
sputtered away. However, the sputtering might then also lead to altered electric fields, especially at sharper edges near heterogeneities (rather than idealized field lines parallel to the sample surface). In such an example, a greater number of species likely would impinge on the surface at an angle other than perpendicular, which would cause some parts of the material to sputter at different rates (as described with additional theory below (cf. Figure 3)). At these large dimensions, evolved topography would be smoothed at the transition between adjacent materials; experimental evidence is given later. Relatively large surface heterogeneities are most relevant to glow discharge surfaceimaging techniques ${ }^{17-20}$, which typically offer spatial resolution on the scale of hundreds of micrometers.

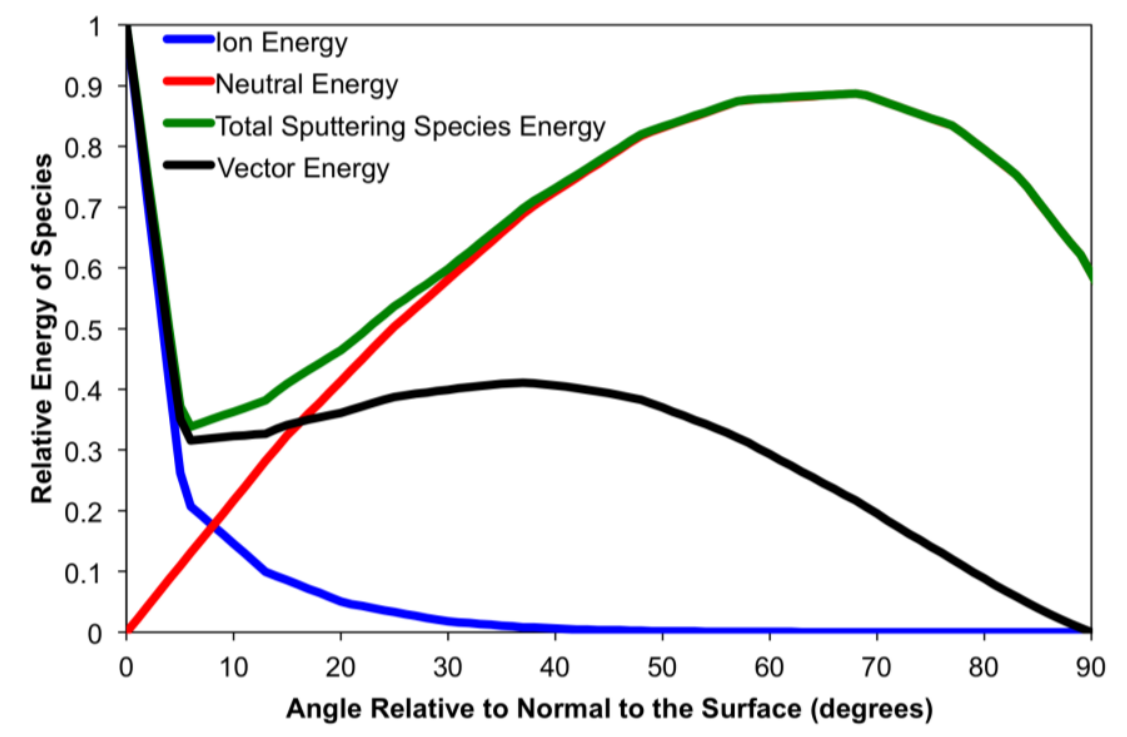

Figure 3: Neutral and ion energies related to the angle at which species impinge upon a sample surface. Total energy distribution is included along with the vector energy distribution (portion of the energy of an incident species directed perpendicular to the surface). 
Surface mixing and redeposition have much different effects when sample heterogeneity is larger in scale. In the case of surface mixing, most laterally adjacent atoms will be the same species. Thus, any rearrangement on the surface will have little net effect, so the signal and related sputtering rate should remain representative of the localized region. Redeposition has been studied experimentally by means of a large heterogeneity (a copper insertion) in an aluminum substrate). ${ }^{33}$ In those experiments, sputtered copper from the inclusion redeposited across the sample and in time an equilibrium was established between redeposited and resputtered atoms. However, this equilibrium then did not accurately reflect the true elemental composition of the surface.

Finally, there is no guarantee that plasma conditions will be consistent during elemental analysis of a sample by imaging GD spectrometry. As described by Voronov and coworkers ${ }^{26}$, large samples and elevated pressures used for such applications are prone to unusual spot patterns in the plasma. These structures could arise in the presence of heterogeneities or lead to unexpected surface morphology.

\subsection{Effect of Directionality of Impinging Species on Sputtering of Heterogeneous Samples}

Theoretical descriptions of glow discharge sputtering of homogeneous materials have included the flux and angular distribution of incident neutral atoms and ions. Figure 3 is derived from these simulations. ${ }^{32,38,39}$ Angular variations 
reveal the effect of sputtering species that do not impinge perpendicularly on the sample surface. Bogaerts and coworkers ${ }^{32}$ described the contribution of neutrals and ions to the sputtering of a flat, uniform cathode. When they accounted for elastic scattering of neutrals, the relative contribution of those neutrals to sputtering dropped due to a wider distribution of incident angles. Figure 3 was compiled by multiplying the calculated mean energies of species moving at a given angle ${ }^{38}$ by their relative population distribution at the cathode ${ }^{32}$ and normalizing these relative energies.

The presence of structure on the bottom of an evolved sputtering crater should have little effect on the contribution of impinging species that approach from an angle normal or nearly normal to the surface, except for those very near a higher adjacent zone that are shaded from ions or neutrals bombarding from sharp angles. However, for surfaces with appreciable topographical variation, non-perpendicular species might impinge upon the side of an evolved feature and thereby induce greater sputtering. Moreover, the greater solid angle accessible to an elevated zone of a sample will result in a greater flux of incident ions and fast neutrals that impact the surface. The result is that transitions between materials will not be sharp-edged; the lateral transition is smoothed as sputtering progresses. Such implications are demonstrated in the experimental results that follow. 


\section{Experimental Results and Discussion}

\subsection{Macroscopic Inclusions}

Much of the theoretical discussion above can be directly verified experimentally for macroscopically heterogeneous materials. The difference in sputtering rates and related behavior for components of a heterogeneous sample is shown in Figure $4 \mathrm{~A}$, where silver and brass portions of a sample are found at the edge of a glow-discharge crater. In this example, the crater becomes deeper for more than the first $100 \mu \mathrm{m}$ from the crater edge for both brass and the fastersputtering silver, as shown in Figure 4B. Though the difference in sputtering rates diverges from the very edge of the crater, the difference in depth is greatest in the flat portion of each crater. 


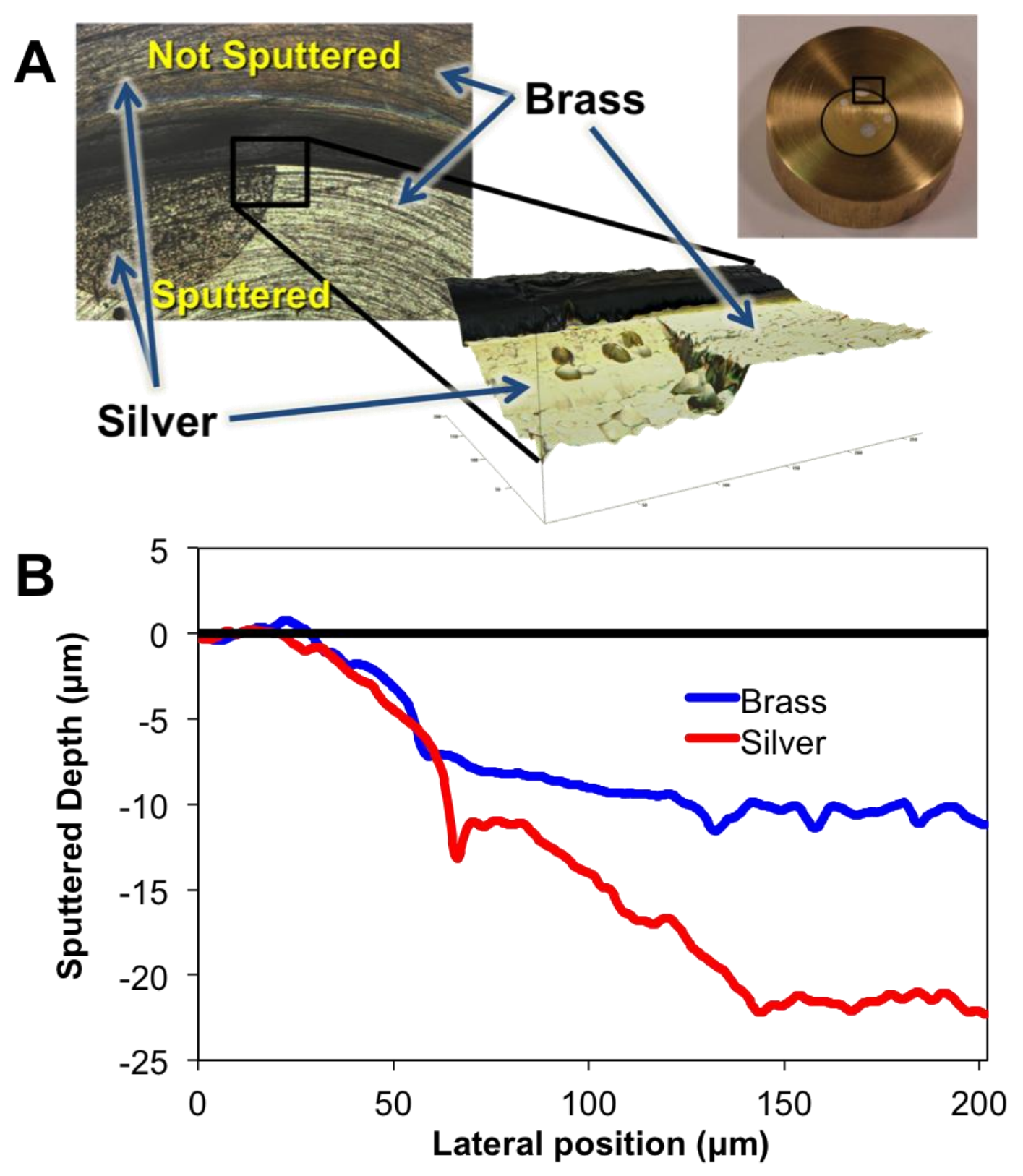

Figure 4: Silver inclusion at the edge of a mostly brass sample. (A) As imaged with a profiling microscope after four hours of GD sputtering. (B) Line profiles of each material show differences in the transition from the edge to the bottom of the crater for each material. 


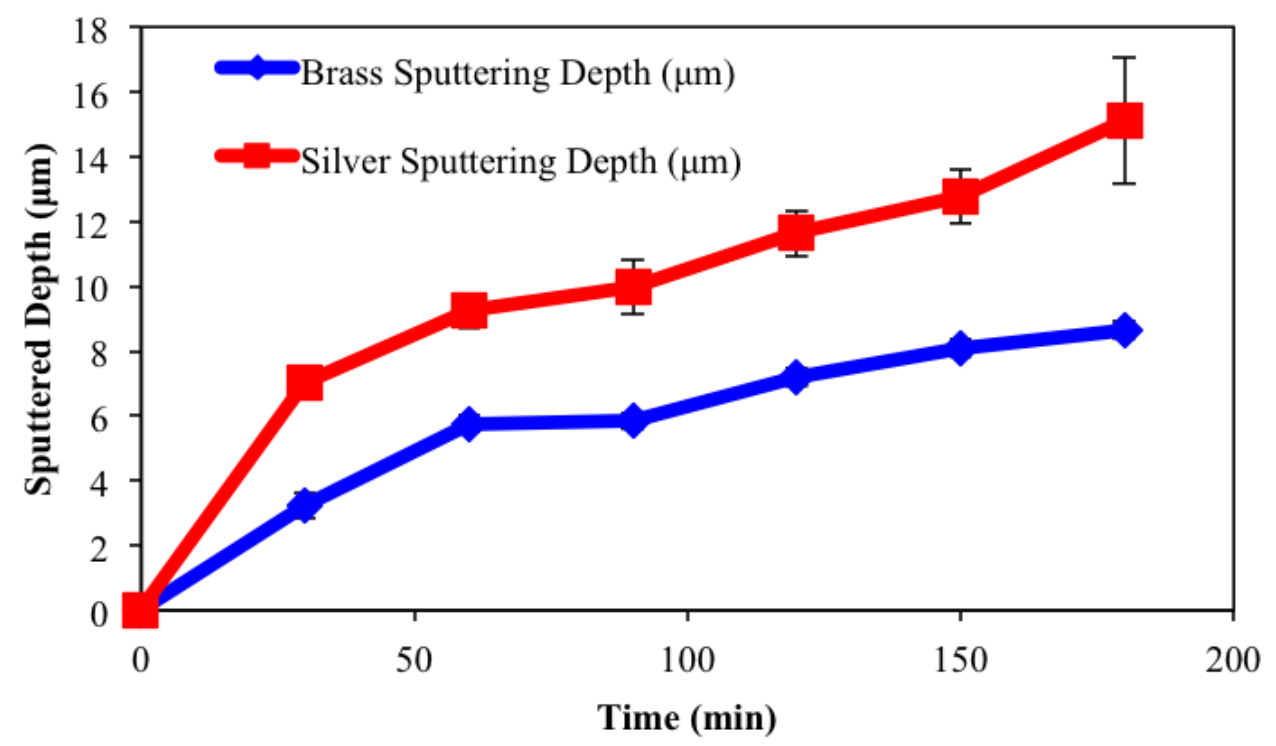

Figure 5: Sputtering depth of adjacent materials as a function of time. The same sample shown in Figure 4 was removed after each sequential time interval; the silver inclusion was centered in the discharge for these experiments.

The sputtering rate (cf. Figure 5) underscores the disparity in sputtered depth between brass and silver and shows that it is greatest during the first period of the sputtering process. When the sample was repeatedly removed from the discharge, measured, and remounted, the relative depth of the silver and brass changed substantially between only the first two measurements. After that time, the two traces in Figure 5 move approximately parallel to one another. The emission behavior of alloys has been shown to equilibrate similarly in other studies. $^{8,40}$ Just as an equilibrium in surface concentration is established for materials with microscopic heterogeneity (cf. Figure 2), equilibration of the sputtering rate can develop also for larger heterogeneities when the bulk and the inclusion have different initial sputtering rates. This behavior departs significantly 
from the generalized model of Payling ${ }^{6}$, insofar as the change in sputtering rate is not necessarily constant across the surface; some areas experience slowing in erosion rather than acceleration. Accordingly, when heterogeneity exists, the sputtering rate might slow in certain areas of the sample. This behavior is not entirely surprising, as sputtering rate most closely aligns with concentration at the surface rather than the bulk. ${ }^{41}$ Enrichment of slower-sputtering material from one region could also retard the sputtering rate elsewhere because of redeposition of the slower-sputtering species. ${ }^{33}$

In Figure 4A, the edge of the brass zone is rounded where it meets the silver. At sharp edges, an elevated electric field could emerge and attract a greater solid angle of impinging and emerging species, as described above with respect to Figures 2 and 3 . As the difference in solid angle develops at a lateral interface, more species can impinge on the newly formed edge to erode it.

Two- and three-dimensional renderings of eroded copper and aluminum inclusions in brass are shown in Figures 6A-D. Figures $6 \mathrm{~A}$ and $6 \mathrm{~B}$ show a slower-sputtering aluminum pin (gray) in a brass sample (green). In this case, the aluminum is rounded near the brass as evidenced in Figure 6A. Copper (brown) and brass (green), shown in Figures 6C and 6D, have sufficiently similar sputtering rates that only a minor difference in depth is apparent. However, the point where the materials meet includes a narrow gap between the copper pin and the brass and rounding on each material at distances away from the gap. Both features developed during the sputtering process. 


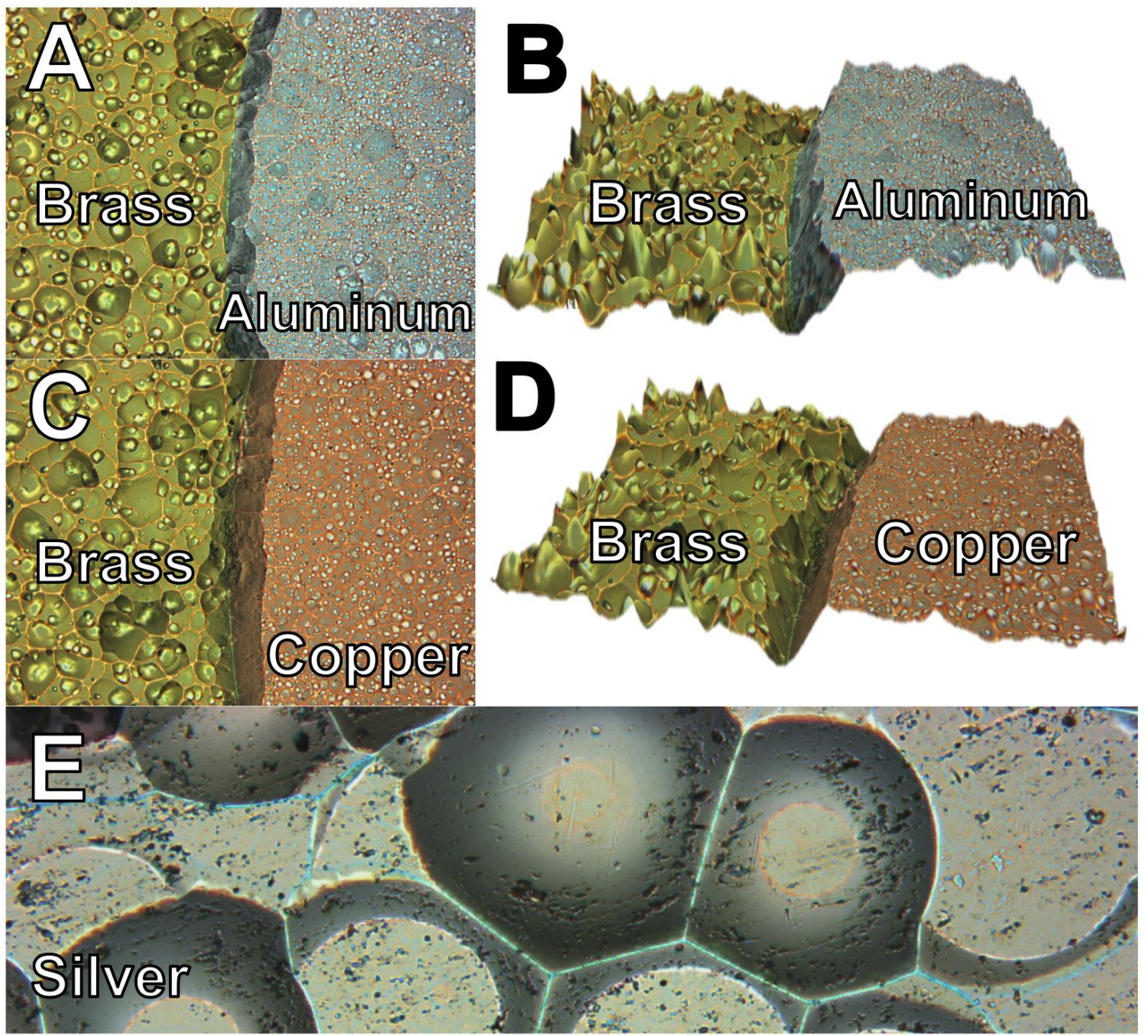

Figure 6: True-color images of the interface between brass and aluminum in two (A) and three (B) dimensions and between brass and copper (C and D). The observed field of view for each of these images was $203 \mu \mathrm{m} \times 270 \mu \mathrm{m}$. The mean step height in B and D was $2.9 \mu \mathrm{m}$ and $1.2 \mu \mathrm{m}$, respectively (brass is lower in both cases). A gap that developed during sputtering between the brass and copper in D extends another $2 \mu \mathrm{m}$ deeper. Microscopic heterogeneity is shown also for a silver sample with a field of view of $85 \mu \mathrm{m} \times 270 \mu \mathrm{m}(\mathrm{E})$. All images show the surface after two hours of GD sputtering. 
Figure 6B shows granular structure evident in an alloy (brass) that can emerge through the sputtering process. This granularity is not due simply to the alloying of the material; the silver surface (which comes from a $99 \%$ pure silver wire) shown in Figure 6E also shows a very granular structure that appears to be comprised of large spherical particles of silver within the Ag wire inclusion.

\subsection{Effects of Surface Morphology in a Homogeneous Material}

Figures $7 \mathrm{~A}$ and $7 \mathrm{~B}$ show a mechanical scratch on a brass sample before and after two hours of sputtering, respectively. Sputtering clearly alters the depth and shape of the scratch such that transitions between high and low surfaces, even when comprised of the same material, are softened; furthermore, the smaller features in the bottom of the scratch disappear. Clearly, the surface is not being eroded evenly, emphasizing the importance of surface finish and preparation in conventional glow-discharge spectrometry. Moreover, a situation similar to that depicted in Figure 7A could develop in a sample after sputtering through an inclusion, leaving a depressed or elevated region of the sample. Here, even after extensive sputtering of the surface (cf. Figure 7B), a difference in height between the bottom of the scratch and the rest of the surface persists, despite smoothing and a reduction in the magnitude of the depression. In depthprofiling analysis, this variation in height could propagate through the measurement. 

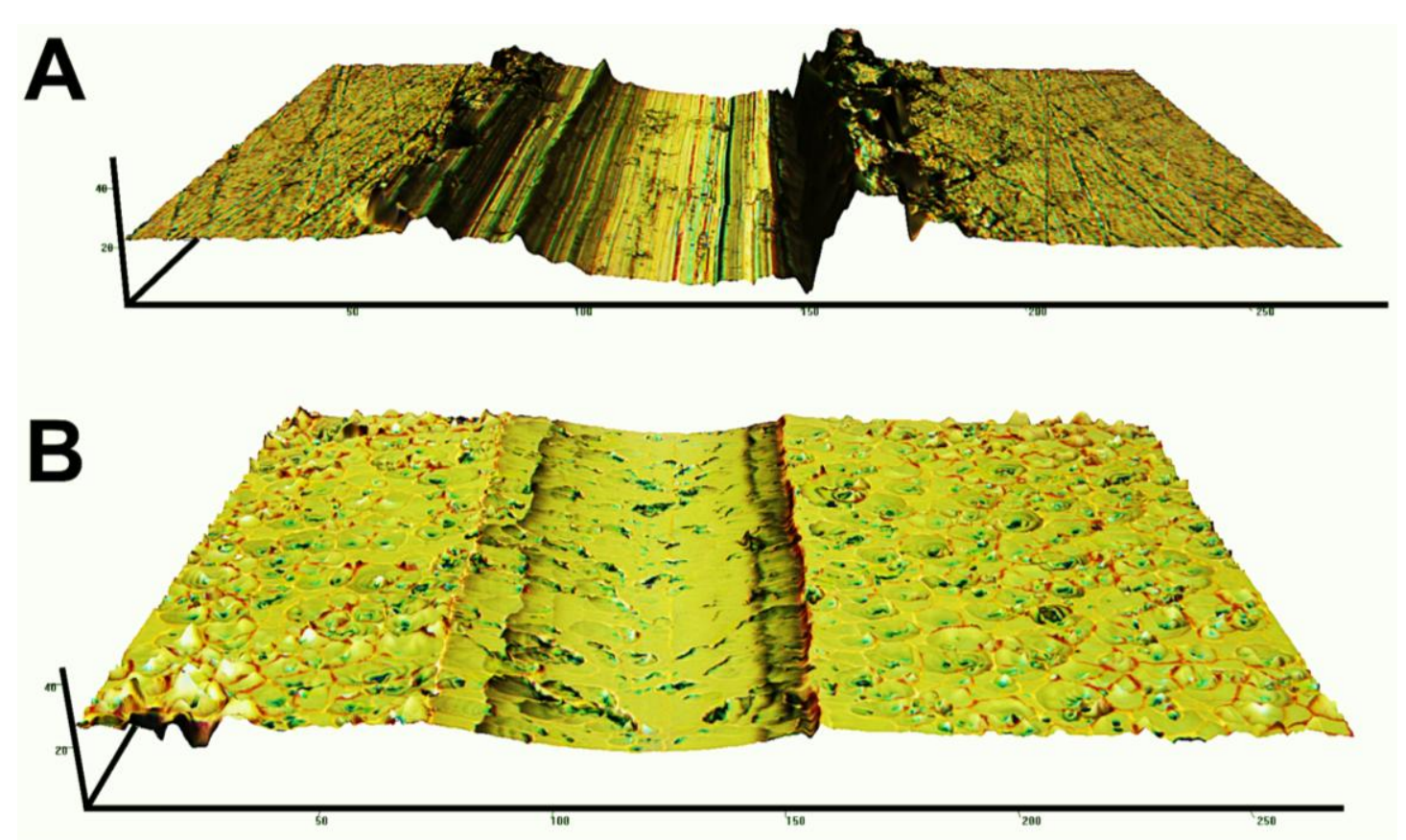

Figure 7: Scratch on a brass sample before $(A)$ and after $(B)$ two hours of glow discharge sputtering. The full horizontal axis of each image is $270 \mu \mathrm{m}$ and the full vertical axis is $45 \mu \mathrm{m}$.

From Figure 7, sputtering rate across a sample results not only from differences in materials present at the surface, but also from surface topography. Therefore, the use of composition alone for determining sputtering rates and quantitation is questionable; surface morphology should also be considered. This deviation of experimental results from commonly applied theory illuminates the challenges that exist for the characterization of heterogeneous samples by GD spectrometry. 


\section{Conclusion}

Many challenges remain for analysis of heterogeneous-surface samples by GD spectrometry. Current theory for quantitation assumes lateral homogeneity for depth profiling and macroscopic homogeneity for bulk analysis, with limited capability to flag the existence of heterogeneous inclusions. Recently, imaging tools have demonstrated the ability to recognize heterogeneity and offer a potential solution to this persistent problem. However, even knowledge of the lateral elemental distribution cannot solve the problem for three-dimensional profiling with GD spectrometry without a more general theory of how sputtering propagates through a heterogeneous sample. Several kinds of heterogeneity have been demonstrated in the present work to exhibit complex behavior when examined after sputtering in a glow discharge.

Disparity in sputtering rates produces additional and significant challenges for imaging GD spectrometry that aims to achieve acceptable depth precision and accuracy. Because current instruments lack the ability to measure both lateral dimensions and a spectrum at the same time, depth resolution would be diminished greatly if elements (or their lateral distribution) must be measured sequentially. The depth to which each measurement corresponds would change as the spectrometer is scanned between elements of interest. Even if the lateral and spectral dimensions would be measured simultaneously, surface topography that exists or emerges could propagate through the data for all layers of a sample. Clearly, additional studies of how sputtering progresses in heterogeneous 
samples are needed before three-dimensional profiling with glow discharge spectrometry can be reliably achieved.

\section{Acknowledgements}

Supported in part by the United States Department of Energy through grant DEFG02-98ER 14890.

\section{References}

1. Bengtson, A.; Danielsson, L., Depth profiling of thin films using a Grimm-type glow discharge lamp. Thin Solid Films 1985, 124 (3), 231-236.

2. Dharmadasa, I. M.; Ives, M.; Brooks, J. S.; France, G. H.; Brown, S. J., Application of glow discharge optical emission spectroscopy to study semiconductors and semiconductor devices. Semicond. Sci. Technol. 1995, 10 (3), 369.

3. Pisonero, J., Glow discharge spectroscopy for depth profile analysis: from micrometer to sub-nanometer layers. Anal. Bioanal. Chem. 2006, 384 (1), 47-49. 4. Boumans, P. W. J. M., Sputtering in a glow discharge for spectrochemical analysis. Anal. Chem. 1972, 44 (7), 1219-1228.

5. Bengtson, A., A contribution to the solution of the problem of quantification in surface analysis work using glow discharge atomic emission spectroscopy. Spectrochim. Acta, Part B 1985, 40 (4), 631-639.

6. Payling, R., Boumans' equation revisited. Part II: A new sputtering equation. Surf. Interface Anal. 1994, 21 (11), 791-799.

7. Dogan, M.; Laqua, K.; Massmann, H., Spektrochemische Analysen mit einer Glimmentladungslampe als Lichtquelle-I. Spectrochim. Acta, Part B 1971, 26 (10), 631-649.

8. Weiss, Z., Analysis of graphitized cast irons by optical emission spectroscopy: matrix effects in the glow discharge and the spark excitation. Spectrochim. Acta, Part B 1996, 51 (8), 863-876.

9. Efimova, V.; Hoffmann, V.; Eckert, J., Sputter crater formation in the case of microsecond pulsed glow discharge in a Grimm-type source. Comparison of direct current and radio frequency modes. Spectrochim. Acta, Part B 2012, 76, 181-189.

10. Raith, A.; Hutton, R. C.; Huneke, J. C., Optimization of quantitative depth profiling with glow discharge mass spectrometry. Part 1. Optimization studies on crater shape and time-depth conversion. J. Anal. At. Spectrom. 1993, 8 (6), 867873.

11. Pons-Corbeau, J.; Cazet, J. P.; Moreau, J. P.; Berneron, R.; Charbonnier, J. C., Quantitative surface analysis by glow discharge optical spectrometry. Surf. Interface Anal. 1986, 9 (1), 21-25. 
12. Bengtson, A.; Hänström, S.; Lo Piccolo, E.; Zacchetti, N.; Meilland, R.; Hocquaux, H., Development of a standard method for quantitative depth profile analysis of zinc-based metallic coatings by direct current glow discharge optical emission spectroscopy. Surf. Interface Anal. 1999, 27 (8), 743-752.

13. Weiss, Z., New method of calibration for glow discharge optical emission spectrometry. J. Anal. At. Spectrom. 1994, 9 (3), 351-354.

14. Weiss, Z., Emission yields and the standard model in glow discharge optical emission spectroscopy: Links to the underlying physics and analytical interpretation of the experimental data. Spectrochim. Acta, Part B 2006, 61 (2), 121-133.

15. Hudson, R. M.; Biber, H. E.; Oles, E. J.; Warning, C. J., Surface segregation of manganese in low-carbon steel during annealing. Metall. Trans. A 1976, 7 (12), 1857-1865.

16. Kluken, A. O.; Grong, $\varnothing$., Mechanisms of inclusion formation in Al-Ti-Si-Mn deoxidized steel weld metals. Metall. Trans. A 1989, 20 (8), 1335-1349.

17. Hoffmann, V.; Ehrlich, G., Investigations on the lateral distribution of the emission line intensities in the plasma of a Grimm-type glow discharge source. Spectrochim. Acta, Part B 1995, 50 (4-7), 607-616.

18. Webb, M. R.; Hoffmann, V.; Hieftje, G. M., Surface elemental mapping using glow discharge-optical emission spectrometry. Spectrochim. Acta, Part B 2006, 61 (12), 1279-1284.

19. Gamez, G.; Frey, D.; Michler, J., Push-broom hyperspectral imaging for elemental mapping with glow discharge optical emission spectrometry. J. Anal. At. Spectrom. 2012, 27 (1), 50-55.

20. Voronov, M.; Hoffmann, V.; Wallendorf, T.; Marke, S.; Monch, J.; Engelhard, C.; Buscher, W.; Ray, S. J.; Hieftje, G. M., Glow discharge imaging spectroscopy with a novel acousto-optical imaging spectrometer. J. Anal. At. Spectrom. 2012, 27 (3), 419-425.

21. Matsushita, T.; Guo, F. Z.; Matsui, F.; Kato, Y.; Daimon, H., Threedimensional atomic-arrangement reconstruction from an Auger-electron hologram. Phys. Rev. B: Condens. Matter Mater. Phys. 2007, 75 (8), 085419. 22. Hamedani, H. A.; Allam, N. K.; El-Sayed, M. A.; Khaleel, M. A.; Garmestani, H.; Alamgir, F. M., An Experimental Insight into the Structural and Electronic Characteristics of Strontium-Doped Titanium Dioxide Nanotube Arrays. Adv. Funct. Mater. 2014, 24 (43), 6783-6796.

23. Fisher, G. L.; Belu, A. M.; Mahoney, C. M.; Wormuth, K.; Sanada, N., ThreeDimensional Time-of-Flight Secondary lon Mass Spectrometry Imaging of a Pharmaceutical in a Coronary Stent Coating as a Function of Elution Time. Anal. Chem. 2009, 81 (24), 9930-9940.

24. Nelis, T.; Pallosi, J., Glow discharge as a tool for surface and interface analysis. Appl. Spectrosc. Rev. 2006, 41 (3), 227-258.

25. Winchester, M. R.; Marcus, R. K., Emission characteristics of a pulsed, radiofrequency glow discharge atomic emission device. Anal. Chem. 1992, 64 (18), 2067-2074.

26. Voronov, M.; Hoffmann, V.; Steingrobe, T.; Buscher, W.; Engelhard, C.;

Storey, A. P.; Ray, S. J.; Hieftje, G. M., Spot patterns and instabilities in a pulsed 
low-pressure rf glow discharge. Plasma Sources Sci. Technol. 2014, 23 (5), 054009.

27. Galindo, R. E.; Forniés, E.; Albella, J., Interfacial effects during the analysis of multilayer metal coatings by radio-frequency glow discharge optical emission spectroscopy Part 1. Crater shape and sputtering rate effects. J. Anal. At. Spectrom. 2005, 20 (10), 1108-1115.

28. Coburn, J.; Eckstein, E.; Kay, E., Elemental composition profiling in thin films by glow - discharge mass spectrometry: Depth resolution. J. Appl. Phys. 1975, 46 (7), 2828-2830.

29. Bengtson, A., Quantitative depth profile analysis by glow discharge. Spectrochim. Acta, Part B 1994, 49 (4), 411-429.

30. Präßler, F.; Hoffmann, V.; Schumann, J.; Wetzig, K., Quantitative depth profiling in glow discharge spectroscopies - A new deconvolution technique to separate effects of an uneven erosion crater shape. Fresen. J. Anal. Chem. 1996, 355 (7), 840-846.

31. Heintz, M. J.; Broekaert, J. A. C.; Hieftje, G. M., Analytical characterization of a planar magnetron radio frequency glow-discharge source. Spectrochim. Acta, Part B 1997, 52 (5), 579-591.

32. Bogaerts, A.; Van Straaten, M.; Gijbels, R., Monte Carlo simulation of an analytical glow discharge: motion of electrons, ions and fast neutrals in the cathode dark space. Spectrochim. Acta, Part B 1995, 50 (2), 179-196.

33. Ferreira, N.; Büger, P., Redeposition of Sputtered Material in a Glow-

Discharge Lamp Measured by Means of an Ion Microprobe Mass Analyser. $Z$. Naturforschung, A: Phys. Sci. 1978, 33 (2), 141-144.

34. Ziegler, J. F., Stopping of Energetic Light lons in Elemental Matter. J. Appl. Phys. 1999, 85 (3), 1249-1272.

35. Alvarez-Toral, A.; Sanchez, P.; Menéndez, A.; Pereiro, R.; Sanz-Medel, A.; Fernández, B., Depth Profile Analysis of Amorphous Silicon Thin Film Solar Cells by Pulsed Radiofrequency Glow Discharge Time of Flight Mass Spectrometry. J. Am. Soc. Mass Spectrom. 2015, 26 (2), 305-314.

36. Takahara, H.; Ishigami, R.; Kodama, K.; Kojyo, A.; Nakamura, T.; Oka, Y., Hydrogen analysis in diamond-like carbon by glow discharge optical emission spectroscopy. J. Anal. At. Spectrom. 2016, 31 (4), 940-947.

37. Heikkilä, I.; Eggertson, C.; Randelius, M.; Caddeo-Johansson, S.; Chasoglou, D., First experiences on characterization of surface oxide films in powder particles by Glow Discharge Optical Emission Spectroscopy (GD-OES). Metal Powder Report. 2016, 71 (4), 261-264.

38. Mason, R. S.; Allott, R. M., The theory of cathodic bombardment in a glow discharge by fast neutrals. J. Phys. D: Appl. Phys. 1994, 27 (11), 2372.

39. Wu, C.-k.; International Symposium on Plasma, C., Symposium proceedings/ISPC-13: 13th International Symposium on Plasma Chemistry; August 18-22, 1997; Beijing, China. Suppl.(1997). Peking University Press: 1997. 40. Wagatsuma, K.; Hirokawa, K., Observation of Cu-Ni alloy surfaces by low wattage glow discharge emission spectrometry. Surf. Interface Anal. 1984, 6 (4), 167-170. 
41. Payling, R.; Jones, D. G., Fundamental parameters in quantitative depth profiling and bulk analysis with glow discharge spectrometry. Surf. Interface Anal. 1993, 20 (9), 787-795. 


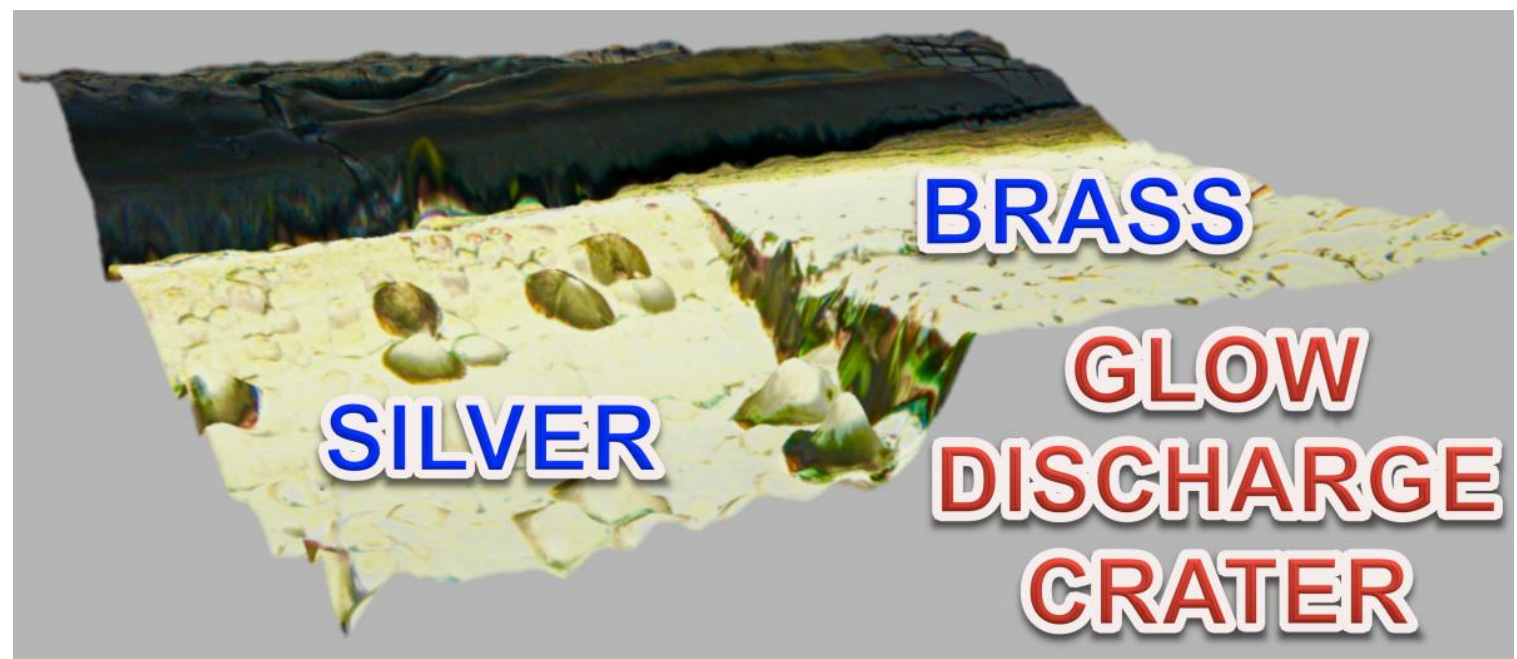

